\title{
CLOUD STORAGE LIMIT MONITORING MEASURES
}

\author{
Jayachandran. $\mathbf{R}^{1}$ \\ ${ }^{I}$ Department of CSE, saveetha school of Engineering, chennai
}

\begin{abstract}
We are well aware that cloud storage is becoming very popular and also in wide use nowadays. it is one of the best infrastructure based service. in this paper, we are going to introduce the measures that can be taken to monitor the cloud storages. this is important because, one should be always aware of the storage status of the cloud. so that he can decide what whether he should get some more space in the cloud or to delete the datum from the existing cloud and remain using the same.
\end{abstract}

This measures also use the mobile smart phones to indicate the status of the cloud storage, so that one need not login to the cloud storage every time, when he want to know the status.

\section{INTRODUCTION}

In this technique we are going to use mobile phones and computers to indicate the storage limit and we also use the effective communication between the phones and the computers connected to the internet.

\section{SMART PHONE}

A smartphone, or smart phone, is a mobile phone with more advanced computing capability and connectivity than basic feature phones.

Early smartphones typically combined the features of a mobile phone with those of another popular consumer device, such as a personal digital assistant (PDA), a media player, adigital camera.

Modern smartphones include all of those features plus the features of a touchscreen computer, including web browsing, Wi-Fi, and 3rd-party apps.

Currently, about $90 \%$ of handset sales worldwide are for devices

\section{CLOUD STORAGE}

Cloud storage is a model of networked enterprise storage where data is stored in virtualized pools of storage which are generally hosted by third parties. Hosting companies operate large data centers, and people who require their data to be hosted buy or lease storage capacity from them. The data center operators, in the background, virtualize the resources according to the requirements of the customer and expose them as storage pools, which the customers can themselves use to store files or data objects. Physically, the resource may span across multiple servers and multiple locations. The safety of the files depends upon the hosting companies, and on the applications that leverage the cloud storage.
Cloud storage services may be accessed through a web service application programming interface (API) or by applications that utilize the API, such as cloud desktop storage, acloud storage gateway or Web-based content management systems.

\section{INTERNET}

The Internet is a global system of interconnected computer networks that use the standard Internet protocol suite (TCP/IP) to link several billion devices worldwide. It is a network of networks that consists of millions of private, public, academic, business, and government networks, of local to global scope, that are linked by a broad array of electronic, wireless, and optical networking technologies. The Internet carries an extensive range of information resources and services, such as the inter-linked hypertextdocuments and applications of the World Wide Web (WWW), the infrastructure to support email, and peer-to-peer networks for file sharing and telephony.

\section{PROCESS}

The process is made very simple here.the process needs the mobile smartphone with the data connection.

A computer with the internet connection (that is used by the user)

The process starts with synchronizing the mobile with the cloud storage.mobile is programmed in such a way that the mobile monitors the storage space of the cloud continuously.

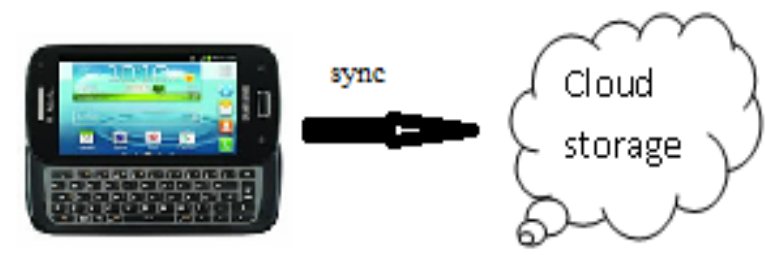


The mobile phone is connected to computer with internet as well so that the mobile can communicate to computer about the status of the cloud storage.

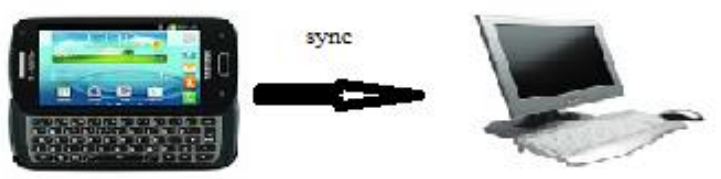

When the cloud storage is full the cloud sends the warning message to the mobile.

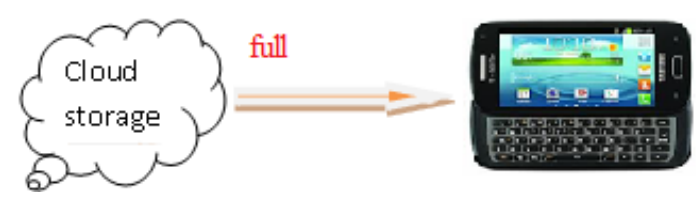

The mobile that is synchronized with the computers communicates to the computers that the storage is full and they cannot update anymore files.

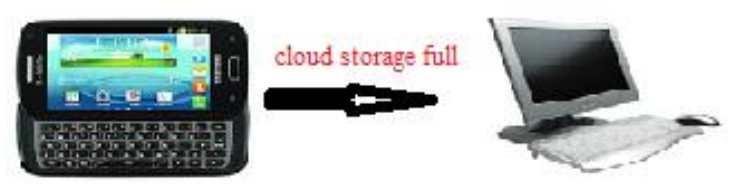

Then the computer starts sending files to the cloud.

\section{THE COMPLETE PROCESS}

The mobile smart phone is connected to the cloud. the smart phone is also connected with the computer with the internet. when the cloud storage is full the cloud sends the warning message that the cloud storage is full. then the mobile sends the error message to the computer. then the computer stops sending the files to the cloud.

\section{CONCLUSIONS}

With the measures introduced in this paper it is very much possible to monitor the cloud storage space and thereby have the idea of the cloud storage limit is filled or not.

\section{REFERENCES}

[1] Z. Wilcox-O'Hearn and B. Warner, "Tahoe: the leastauthority filesystem," in StorageSS, 2008, pp. 21-26. Full Text: Access at ACM

[2] K. D. Bowers, A. Juels, and A. Oprea, "HAIL: A HighAvailability and Integrity Layer for Cloud Storage," in 16th ACM Conference on Computer and Communications Security, CCS '09. ACM, 2009, pp. 187-198. Full Text: Access at ACM

[3] R. A. Popa, J. Lorch, D. Molnar, H. J. Wang, and L. Zhuang, "Enabling Security in Cloud Storage SLAs with CloudProof," in USENIX Annual Technical Conference (USENIX), 2011.

[4] A. Shraer, C. Cachin, A. Cidon, I. Keidar, Y. Michalevsky, and D. Shaket, "Venus: verification for untrusted cloud storage," in CCSW, 2010, pp. 19-30. Full Text: Access at ACM

[5] H. Abu-Libdeh, L. Princehouse, and H. Weatherspoon, "Racs: a case for cloud storage diversity," in 1st ACM Symposium on Cloud Computing, SoCC 2010, 2010, pp. 229-240. Full Text: Access at ACM

[6] E. Stefanov, M. van Dijk, A. Oprea, and A. Juels, "Iris: A scalable cloud file system with efficient integrity checks," in 28th Annual Computer Security Applications Conference (ACSAC 2012). ACM, 2012. [CrossRef]

[7] A. N. Bessani, M. P. Correia, B. Quaresma, F. André, and P. Sousa, "DepSky: Dependable and Secure Storage in a Cloud-of-Clouds," in EuroSys, 2011, pp. 31-46. [CrossRef]

[8] S. Kamara, C. Papamanthou, and T. Roeder, "CS2: A Searchable Cryptographic Cloud Storage System," Microsoft Research, Tech. Rep. MSR-TR-2011-58, 2011.

[9] J. K. Resch and J. S. Plank, "AONT-RS: Blending Security and Performance in Dispersed Storage Systems," in FAST, 2011, pp. 191-202.

[10] J. Spillner, G. Bombach, S. Matthischke, J. Muller, R. Tzschichholz, and A. Schill, "Information Dispersion over Redundant Arrays of Optimal Cloud Storage for Desktop Users," in UCC, 2011, pp. 1-8. Abstract | Full Text: PDF (482KB) |Full Text: HTML

[11] C. Basescu, C. Cachin, I. Eyal, R. Haas, A. Sorniotti, M. Vukolic, and I. Zachevsky, "Robust Data Sharing with Key-Value Stores," in Intl. Conference on Dependable Systems and Networks (DSN). IEEE, 2012. Abstract | Full Text: PDF (376KB) | Full Text: HTML

[12] Amazon Outage, http://www.networkworld.com/news/2012/042712amazon- outage-258735.htmll, Nov. 20. 2012.

[13] Sidekick, http://abcnews.go.com/Business/sidekickdisaster-shows-datas- $\quad$ safe-cloud/story?id=8840420, Nov. 20. 2012.

[14] A. Herzberg, S. Jarecki, H. Krawczyk, and M. Yung, "Proactive Secret Sharing Or: How to Cope With Perpetual Leakage," in CRYPTO, 1995, pp. 339-352. [CrossRef]

[15] A. Shamir, "How to share a secret," Commun. ACM, vol. 22, no. 11, pp. 612-613, 1979. Full Text: Access at ACM

[16] P. Feldman, "A Practical Scheme for Non-interactive Verifiable Secret Sharing," in FOCS, 1987, pp. 427437. Abstract | Full Text: PDF (1714KB)

[17] M. O. Rabin, "Efficient dispersal of information for security, load balancing, and fault tolerance," J. ACM, 
vol. 36, no. 2, pp. 335-348, 1989. Full Text: Access at ACM

[18] H. Krawczyk, "Secret sharing made short," in CRYPTO, 1993, pp. 136-146.

[19] P. Rogaway and M. Bellare, "Robust computational secret sharing and a unified account of classical secretsharing goals," in ACM Conference on Computer and Communications Security, 2007, pp. 172-184.

[20] R. L. Rivest, "All-or-Nothing Encryption and the Package Transform," in Fast Software Encryption (FSE '97), 1997, pp. 210-218. [CrossRef] 